\title{
Puerarin Exerted Anti-Osteoporotic Action Independent of Estrogen Receptor-Mediated Pathway
}

\author{
Seiwa MichiHara, Teruyoshi TanaKa, Yuki UzAwa, Tatsuya MoriYama \\ and Yukio KAWAMURA* \\ Department of Applied Biological Chemistry, Graduate School of Agriculture, Kinki University, \\ Nara 631-8505, Japan
}

(Received December 28, 2011)

\begin{abstract}
Summary Puerarin, a daidzein-8-C glucoside, is the major isoflavonoid in Kudzu (Pueraria lobata), and is unique in that it contains $\mathrm{C}$-C conjugated glucose at position 8 of the isoflavonoid structure. A puerarin diet at a dose of $5 \mathrm{mg} / \mathrm{kg}$ b.w./d to fed ovariectomized mice for 2 mo diminished the urinary deoxypyridinoline, a typical bone-degradation product. Since the bone absorption marker, serum tartarate-resistant acid phosphatase (TRAP) activity of puerarin-fed mice decreased but the bone formation marker, osteocalcin level, did not alter, the puerarin diet was proved to specifically depress the bone absorption, but not the overall bone metabolism. In accordance with that results, the femur structure of puerarin-fed mice was restored compared with that of puerarin-free diet mice. The atrophied uterine due to low estrogen (E2) level after ovariectomy was not restored by the puerarin diet, suggesting that puerarin exerted the anti-osteoporotic action through a non estrogen receptor (ER) mediated-pathway, in vivo. The growth of an ER-positive human breast cancer cell, MCF-70, was not enhanced by puerarin, suggesting that puerarin did not show estrogen-like action on MCF-7 cells, even at a ten thousand times higher concentration than that of E2. Furthermore, ICI182,780 (ICI), an estrogen antagonist, suppressed the enhanced growth of MCF-7 cells by E2, but not that by puerarin. In an ER-binding assay, puerarin was proved not to bind to ER $\alpha$ or $\beta$, or if all, extremely weakly, although daidzein, an aglycon of puerarin, showed a little stronger binding compared with puerarin. All these results strongly indicate that puerarin exerts its anti-osteoprotic action independently of the ERmediated pathway.
\end{abstract}

Key Words puerarin, anti-osteoporosis, estrogen receptor, bone-loss, Kudzu

Osteoporotic bone loss is the result of high bone turnover in which bone absorption outpaces bone deposition (1-5). This imbalance in bone turnover is induced by estrogen (E2) deficiency in menopausal women and female rodents, and can be ameliorated with bioavailable estrogens including selective estrogen receptor (ER) modulators (6). Most estrogen actions are mediated by $\operatorname{ER} \alpha$ and $\beta$, which are ligand-dependent transcriptional regulators. Several isoflavonoids (phytoestrogens) are known to be able to weakly bind to ERs due to their biphenolic structure, which is required for the ligandreceptor association $(7,8)$ (Fig. 1). They can mimic or modulate the actions of endogenous estrogens by acting like partial ER agonists or antagonists depending on the endogenous estrogen status (9).

Soybeans and soybean isoflavonoids have been examined for their effects on bone metabolism (10-13). However, little is known about effects of other isoflavonoid-rich food sources on bone metabolism. Kudzu (Pueraria lobata) is one of the plants belonging to pulses and contains various isoflavonoids. Kudzu plant, how-

\footnotetext{
* To whom correspondence should be addressed.
}

E-mail: ykawamur@nara.kindai.ac.jp ever, has not been utilized much, especially as a food resource. At present, it is limitedly used as an herbal medicine in East Asia and only the root has been traditionally processed to starch in Japan.

In our previous studies, Kudzu root and vine extracts depressed tartarate-resistant acid phosphatase (TRAP) activity, a differentiation marker of the preosteoclast RAW264.7 cell which was stimulated by nuclear factor kappa-B ligand (RANKL) (14). Moreover, a diet containing Kudzu root and vine extracts significantly depressed bone absorption in ovariectomized (OVX) mice (15). Fifty to $70 \%$ of Kudzu vine and root extracts comprise a unique isoflavonoid, puerarin. The $C$-glycoside nature endows it with resistance to intestinal digestion, which differs from the other easily hydrolysable O-glycosides such as daidzin and genistin. Thus, the bone loss preventing action is most attributable to puerarin, although other possibilities can not be excluded.

In the present study, OVX mice were fed a puerarincontaining diet for 2 mo to confirm whether the puerarin diet prevented the bone loss due to ovariectomy and to learn if an ER mediated pathway was responsible for it or not. 


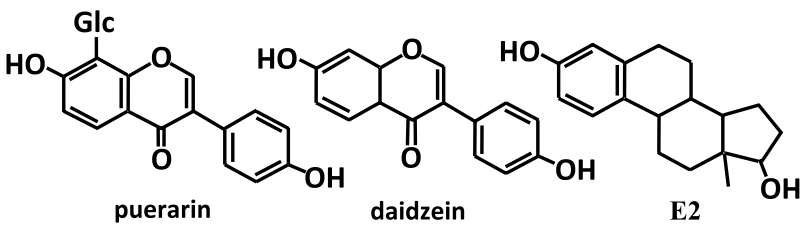

Fig. 1. Chemical structure of puerarin, daidzein, and E2.

\section{MATERIALS AND METHODS}

Materials. Dulbecco's modified Eagle's medium (DMEM) without phenol red was obtained from SigmaAldrich Co. (St. Louis, MO, USA). Fetal bovine serum (FBS) was purchased from Equitech-bio Inc. (Kerrville, TX, USA). Penicillin streptomycin and charcoal-stripped FBS were purchased from Gibco (Carlsbad, CA, USA). Puerarin, daidzein and estrogen (E2) were obtained from Sigma-Aldrich Co. ICI182,780 (ICI), a specific ER inhibitor, was obtained from Tocris Bioscience (Bristol, UK).

Animals and diets. Slc:ddY female mice were purchased from Japan SLC, Inc. (Shizuoka, Japan). They were either ovariectomized (OVX) or Sham-operated (Sham) at $9 \mathrm{wk}$ of age. Sham and OVX mice were divided into the following 4 groups: Sham-control group, Sham-puerarin group, OVX-control group, and OVX-puerarin group. The mice were housed in individual cages under a $12 \mathrm{~h} / 12 \mathrm{~h}$ light/dark cycle at $20 \pm 2{ }^{\circ} \mathrm{C}$. A commercial diet (MF, Oriental Yeast Co., Ltd., Tokyo, Japan) was used as the control diet. The puerarin diet (puerarin content: $5 \mathrm{mg} / \mathrm{kg}$ body weight/ $\mathrm{d}$, approximately $0.165 \mathrm{mg} / \mathrm{animal} / \mathrm{d}$ ) was made by mixing puerarin with MF powder. This dosage was estimated from the puerarin content of Kudzu root or vine in our previous experiment and was below the amount calculated from the daily isoflavonoid-allowance [30$40 \mathrm{mg}$ supplementation/d/man $(60 \mathrm{~kg})]$ recommended in Japan, which corresponded to 0.5 to $0.66 \mathrm{mg} / \mathrm{ani}-$ $\mathrm{mal} / \mathrm{d}$. Because a very small amount of puerarin was added to the fundamental diet, compensation with additional cornstarch was not made. The diet and tap water were supplied ad libitum. Based on these feeding conditions, mice were fed for $8 \mathrm{wk}$. During the experimental period, the body weight and food intake were measured once a week. After $8 \mathrm{wk}$, the blood was taken, and the animals were sacrificed. The heart, liver, spleen, kidney, and uterus were harvested and weighed. The right femur was isolated and ground to analyze the bone intramicrostructure with a microscope. All animals were handled according to International Guiding Principles for Biomedical Research Involving Animals, and this study was approved by the Animal Ethics Committee of Kinki University (approval ID: KAAG-19-003).

Collection of urine and blood samples. Urine was collected directly from the bladder once a week. Blood samples were taken from the tail vein and then centrifuged to collect the serum. Both urine and blood samples were stored at $-80^{\circ} \mathrm{C}$ until further analysis.

Examination of bone absorption markers. Deoxypyr- idinoline (DPD) level in urine was measured using a commercial kit (DS Pharma Biomedical, Osaka, Japan). Urinary creatinine was measured by the picric acid method (Commercial kit from R\&D Systems, MN, USA). The DPD levels are expressed as the ratio of urinary $\mathrm{DPD}$ to the creatinine (Cre) levels (DPD/Cre, nM/ $\mathrm{mM} \cdot \mathrm{Cre})$.

Tartarate resistant acid phosphatase (TRAP) activity. Serum TRAP was measured by the colorimetric method. Briefly, $10 \mu \mathrm{L}$ of serum was added to $80 \mu \mathrm{L}$ of $5 \mathrm{~mm}$ p-nitrophenylphosphate in $50 \mathrm{~mm}$ citric acid buffer, $\mathrm{pH} 4.6$, and incubated for $60 \mathrm{~min}$ at $37^{\circ} \mathrm{C}$. The reaction was stopped by adding $160 \mu \mathrm{L}$ of $0.1 \mathrm{~N} \mathrm{NaOH}$, and the absorbance at $405 \mathrm{~nm}$ was measured with a spectrophotometer (U-0080D, HITACHI, Tokyo, Japan). The TRAP activity was normalized to the serum protein level (U/ $\mu$ g protein). The protein level in each sample was determined by Bradford assay using a protein assay kit (Bio-Rad Laboratories, CA, USA). One unit of enzyme activity is defined as that which hydrolyzes $1 \mu \mathrm{mol}$ of substrate per minute at $37^{\circ} \mathrm{C}$.

Examination of a bone formation marker. Serum osteocalcin concentration was determined with a mouse sandwich ELISA kit (Biomedical Technologies Inc., Osaka, Japan) according to the manufacturer's instructions.

Stereoscopic observation of femoral trabeculae. The distal portions of the isolated right femur were ground with a whetstone. The produced pulverized preparation was observed with a stereoscopic microscope (SZ61, Olympus, Tokyo, Japan).

Effects of puerarin diet on uterine weight and morphology. A hypertrophy test was performed to examine whether or not 8-wk feeding of the puerarin diet exerted estrogenic action in vivo. To examine whether the ddy-strain OVX mice respond to E2, another group of OXV-control mice of the same age (OVX-E2 group) received the subcutaneous injection of E2 $(50 \mu \mathrm{g} / \mathrm{kg}$ b.w./d) twice a week for 2 wk since the oral administration was not effective. After 8-wk feeding with and without puerarin or after the last $2 \mathrm{wk}$ with the E2 injection, mice were sacrificed and the uterus with the oviduct was isolated. The weight of uterus with oviduct was directly measured on a scale. The morphological change was studied with a digital camera connected with a personal computer.

Cell culture. The human breast adenocarcinoma cell line (MCF-7) was obtained from Osaka Bioscience Institute, Japan. MCF-7 cells were maintained in phenol red-free DMEM, containing 10\% (v/v) FBS, penicillin G and streptomycin, and incubated at $37^{\circ} \mathrm{C}$ in $5 \% \mathrm{CO}_{2}$. When the effects of isoflavonoids and E2 were examined, the culture medium was prepared with phenol red-free DMEM containing 10\% (v/v) charcoal-stripped FBS, penicillin G, and streptomycin.

Cell proliferation assay. Cell proliferation was measured by the colorimetric MTT \{[3-(4,5-dimethyl-thiazol-2-yl)-2,5-diphenyltetrazolium bromide] (Dojin, Tokyo, Japan)\} assay. Briefly, acid-isopropanol (0.04 N $\mathrm{HCl}$ in isopropanol) and 3\% sodium lauryl sulfate were 
added to dissolve the reduced MTT crystals (formazan) formed in the cells. After mixing, the absorbance of each well was measured at a wavelength of $595 \mathrm{~nm}$ using $655 \mathrm{~nm}$ as the reference by a microplate reader (BIORAD Model550). For MCF-7 cell growth study, the cells were planted in 96-well plates at a concentration of $2 \times 10^{3}$ cells/well in the medium. One day before starting the experiment, the cells were treated with ICI, E2 $(10 \mathrm{nM})$ and puerarin which were dissolved in dimethyl sulfoxide (DMSO). The final concentration of DMSO in the medium was $0.2 \%$. After cultivation for $4 \mathrm{~d}$, MTT solution was added to the wells ( $50 \mathrm{ng} /$ well) and the plates were incubated for $4 \mathrm{~h}$ followed by the colorimetric determination. To examine the estrogenlike action of puerarin, daidzein and E2, MCF-7 cells were exposed to them in the presence or absence of estrogen antagonist ICI for $4 \mathrm{~d}$ before the growth measurement. Results are expressed as a percentage of control.

Binding assay of puerarin, daidzein, and E2 to ER $\alpha$ and $E R \beta$. The binding of puerarin, daidzein, and E2 was measured by using EnBio ER $(\alpha, \beta) /$ Coactivator, Ligand Assay system (EnBioTec Laboratories, Tokyo, Japan) according to the protocol of the manufacturer. Briefly, the assay sample and recombinant ER were incubated in an avidin-coated 96-well microtiter plate, and the complex between ER and the ligand in the sample bound to the coactivator on the plate was detected by horse radish peroxydase (HRP)-labeled anti-ER antibody. The HRP activity was determined by the tetramethylbenzidine (TMB) method. The reaction was stopped by addition of an acid solution and the resultant color was read at $450 \mathrm{~nm}$ using a microtiter plate spectrophotometer.

Statistical analysis. Data were expressed as means \pm $\mathrm{SE}$, and $n$ denotes the number of replications for each data point. Comparisons of parameters among different groups were made with one-way analysis of variance, followed by the Student-Newman-Keuls multiple comparisons test. In all cases, differences between treatment groups were considered significant at $p<0.05$. All statistical analysis were performed by using Graph Pad Prism 4.02 for Windows.

\section{RESULTS}

Effects of puerarin diet on body weight and food intake

Ten-week-old mice of the Sham-group, Sham-puerarin group, OVX-group, and OVX-puerarin group were fed for $8 \mathrm{wk}$. The effects of puerarin diets $(5 \mathrm{mg} / \mathrm{kg}$ b.w./ d) on the body weight and food intake are shown in Fig. 2. The body weight of each of the four groups did not change during the experiment period of $8 \mathrm{wk}$. The body weight of the OVX-control groups was a little greater than that of the other groups. The body weight of OVXpuerarin group was similar to that of that of Sham groups, but OVX-puerarin group consumed a smaller amount of feed compared with the other groups.

Change in urinary bone absorption markers

As shown in Fig. 2C, a urinary bone metabolic marker, DPD, was increased in the OVX-control group,
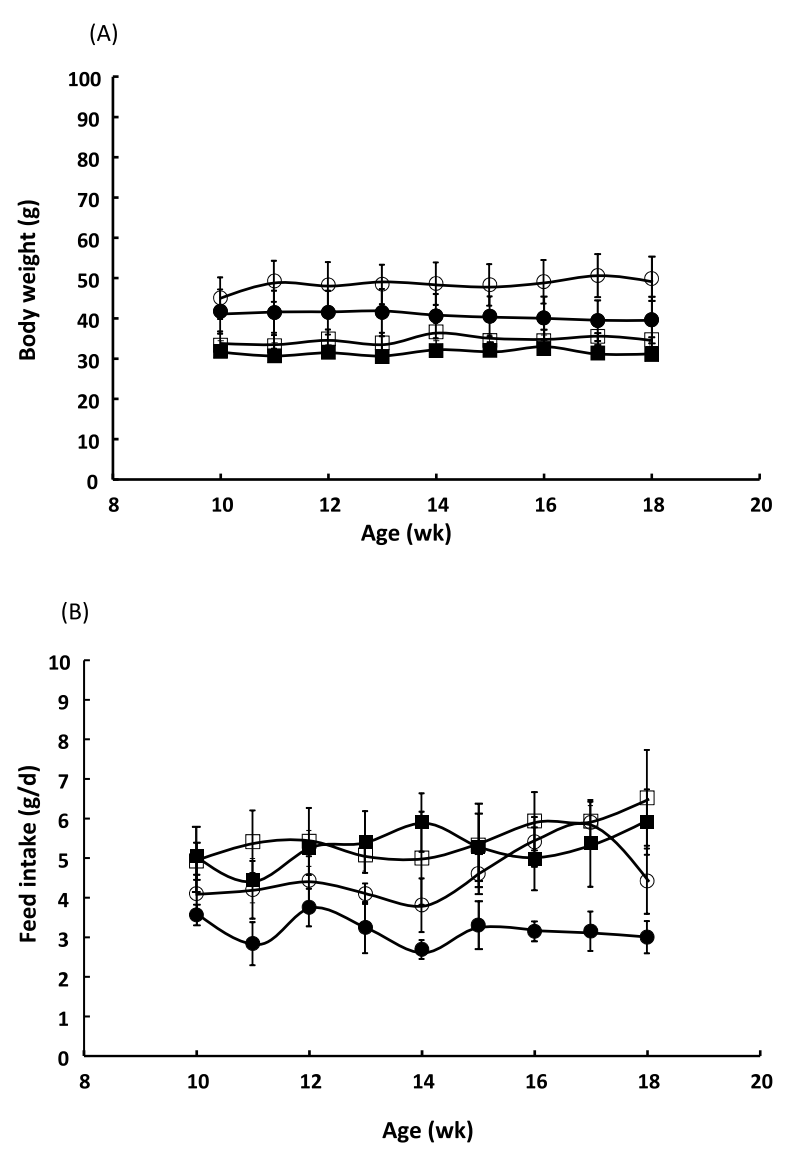

(C)

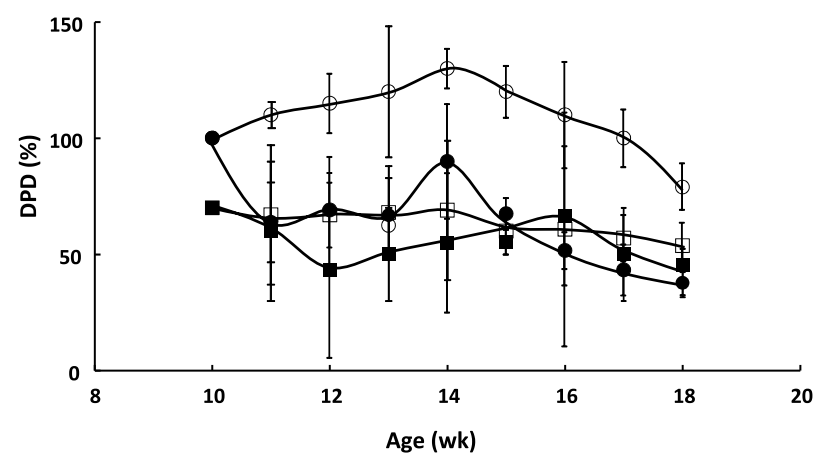

Fig. 2. Effect of puerarin diet on body weight and feed intake, and changes of bone metabolic markers. Sham and OVX mice were divided into the following 4 groups: Sham-control group, Sham-puerarin group, OVX-control group, and OVX-puerarin group. Mice were fed for $8 \mathrm{wk}$ with and without puerarin $(5 \mathrm{mg} / \mathrm{kg}$ body weight/d). During 8 wk, the body weight (A) and food intake (B) were measured once a week. Urine was collected once a week for measurement of DPD (C). Open square: Sham-control group, closed square: Shampuerarin group, open circles: OVX-control group, closed circles: OVX-puerarin group.

whereas in the OVX-puerarin group the marker rapidly decreased during the first week of the feeding to a level similar to those in the Sham groups as observed in our previous study, where the Kudzu root ethanol extract (PREE) was fed (14). On the other hand, there was no change in the Sham mice groups either in the presence 
(A) OVX control

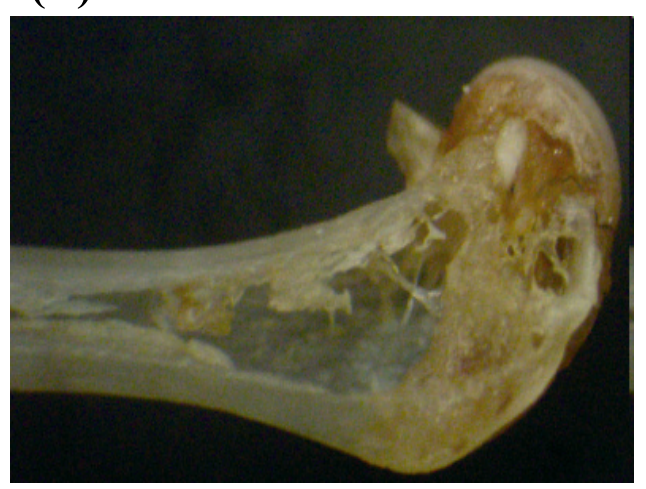

(C) Sham control

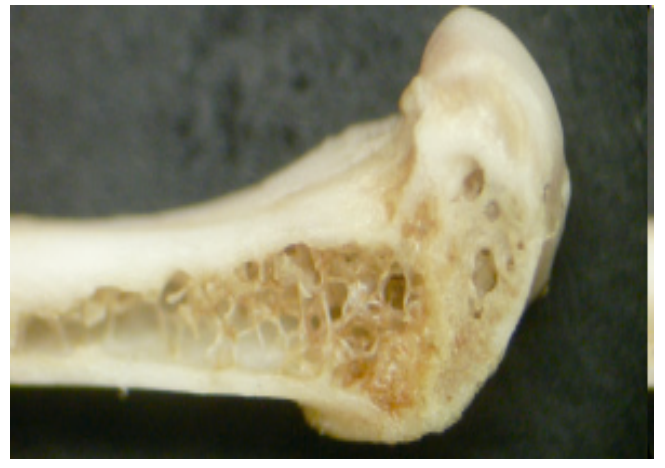

(B) OVX puerarin

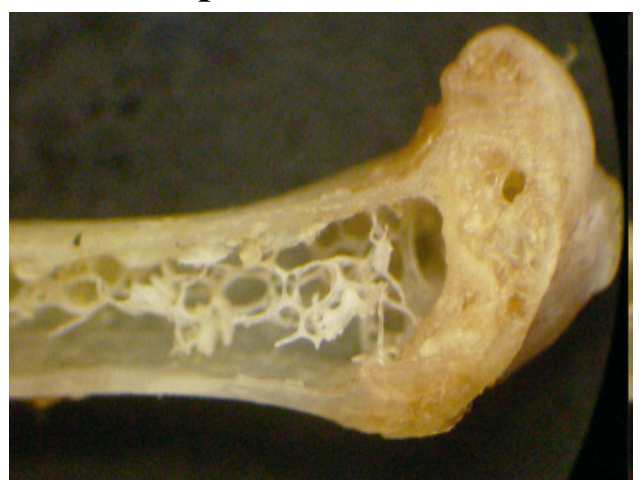

(D) Sham puerarin

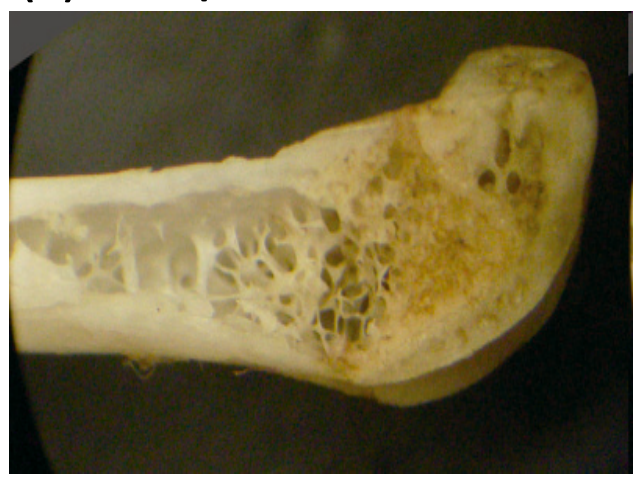

Fig. 3. Stereoscopic observation of femoral trabeculae. After 8 -wk feeding, mice were sacrificed and the right femur was isolated. The distal portions of the isolated right femur were ground with a whetstone. The produced pulverized preparation was observed with a stereoscopic microscope. The upper picture: OVX control mouse (A) and OVX-puerarin mouse (B); the bottom picture: Sham control mouse (C) and Sham-puerarin mouse (D).

(A)

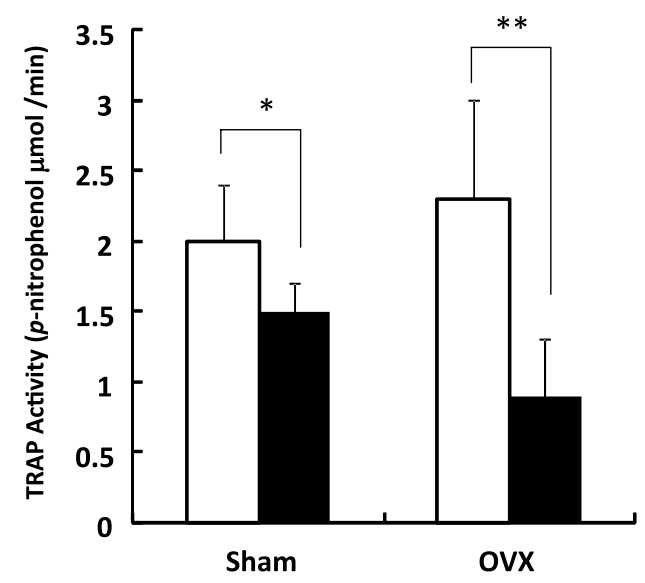

(B)

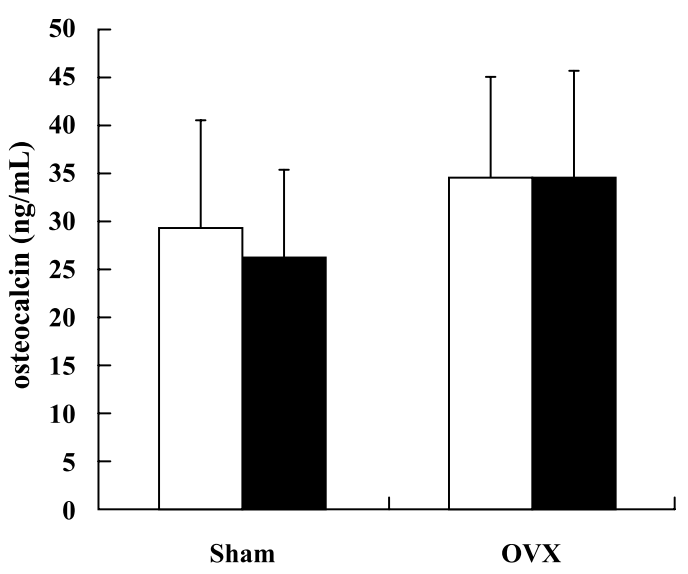

Fig. 4. Effects of puerarin diet on bone absorption and formation marker. After feeding for 8 wk with and without puerarin, blood was collected from the tail vein of OVX and Sham mice. The serum TRAP activity (A) and osteocalcin (B) were determined. White: control group, Black: puerarin group. ${ }^{* *}$ Significant difference from the control $(p<0.01)$. ${ }^{*}$ Significant difference from the control group $(p<0.05)$.

or absence of puerarin. These decreases of final bone degradation products in urine from the puerarin diet suggested that puerarin retarded the bone absorption as the PREE diet did (14).

Effects of puerarin diet on the trabeculae of the femur

To confirm the bone loss-preventing action of dietary puerarin, the femoral trabeculae microstructure after the 8-wk feeding was examined with a stereoscopic microscope. Isolated right femurs were ground with a whetstone. The longitudinal cross sections of the ground femur samples are shown in Fig. 3. The number of femur trabeculae was well maintained in both Sham 


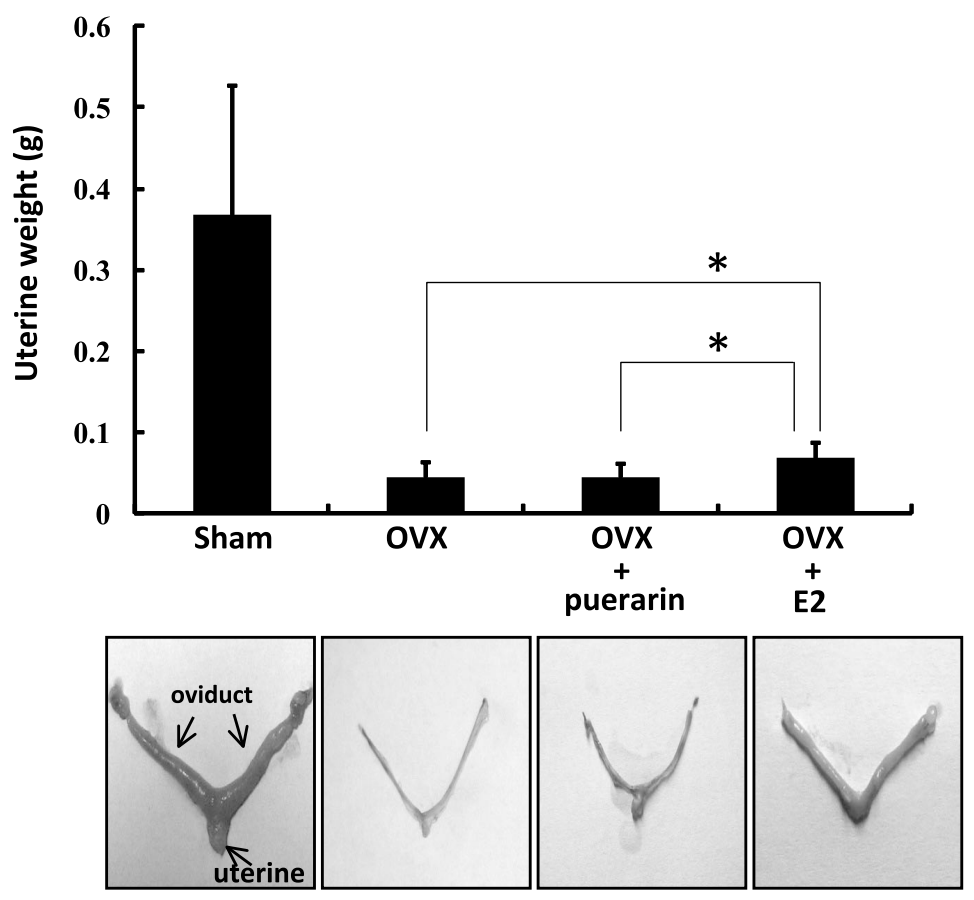

Fig. 5. Effects of puerarin diet on uterine weight and morphology. After feeding for 8 wk with and without puerarin, and in the E2 treatment group (OVX-E2 group) after $2 \mathrm{wk}$ of the injection during the last $2 \mathrm{wk}$ of the experimental period, mice were sacrificed and the uterus with oviduct was isolated. The uterine weight was directly measured on a scale. The morphological change was captured with a personal computer equipped a digital camera. Asterisk indicates significant difference from OVX+E2 group $(p<0.01)$.

groups (Fig. 3C and D). However, the number of trabeculae in the OVX-control group (Fig. 3A) significantly decreased compared with those in both Sham groups. This drastic trabecular destruction in the OVX-control group was improved by the consumption of puerarin in the OVX-puerarin group (Fig. 3B). These observations indicated that the puerarin diet protected the trabecular structure from degradation by osteoclast.

Effects of puerarin diet on bone absorption and formation markers

To confirm whether a puerarin diet affects the bone absorption or bone formation of the mice, a bone absorption marker, serum TRAP activity, and a bone formation marker, osteocalcin, were determined after 8wk feeding with and without puerarin. The puerarin diet suppressed the serum TRAP activity of the OVXpuerarin group more significantly than that of the Sham group as shown in Fig. 4A, On the other hand, the osteocalcin level in the serum did not alter in the puerarin or control groups of both Sham and OVX mice (Fig. 4B). Accordingly, these results indicated that puerarin affected the bone absorption but not the bone formation, presumably by suppressing osteoclast differentiation or maturation.

Effects of puerarin diet on uterine weight and morphology

The uterine and oviduct hypertrophy test was performed to examine whether 8 -wk feeding of the puerarin diet exerted an estrogenic action in vivo, or not. The effects of the puerarin diet on the uterine and oviduct weight and morphology are shown in Fig. 5. The uterine and oviduct weight drastically decreased in OVX mice compared with that of Sham mice. The puerarin

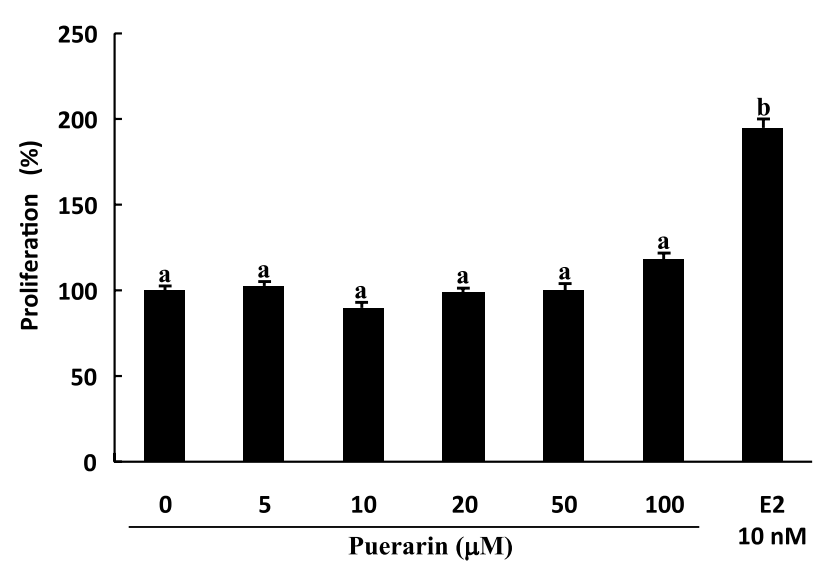

Fig. 6. No estrogen-like action of puerarin on MCF-7 cell proliferation. Hyper-proliferative action of isoflavonoids and E2 on MCF7 cell were examined in the culture medium prepared with phenol red-free DMEM containing $10 \%(\mathrm{v} / \mathrm{v})$ charcoal-stripped FBS. MCF-7 cells were planted in 96-well plates at a concentration of $2 \times 10^{3}$ cells/well. Then, they were exposed to puerarin or E2 for $12 \mathrm{~h}$ and cultivated for $72 \mathrm{~h}$ to check their estrogen responsiveness. The puerarin concentration was raised to $100 \mu \mathrm{M}$. The concentration of E2 was $10 \mathrm{~nm}$ in the medium. $\mathrm{a}, \mathrm{b}<0.01$.

diet fed to OVX mice for $8 \mathrm{wk}$ did not restore their weight. In addition, no hypertrophic change of the morphology was observed in the uterus or oviduct of mice fed the puerarin diet, whereas in mice receiving subcutaneous injection of $\mathrm{E} 2$, the hypertrophic change and weight gain of the uterus and oviduct were observed. 


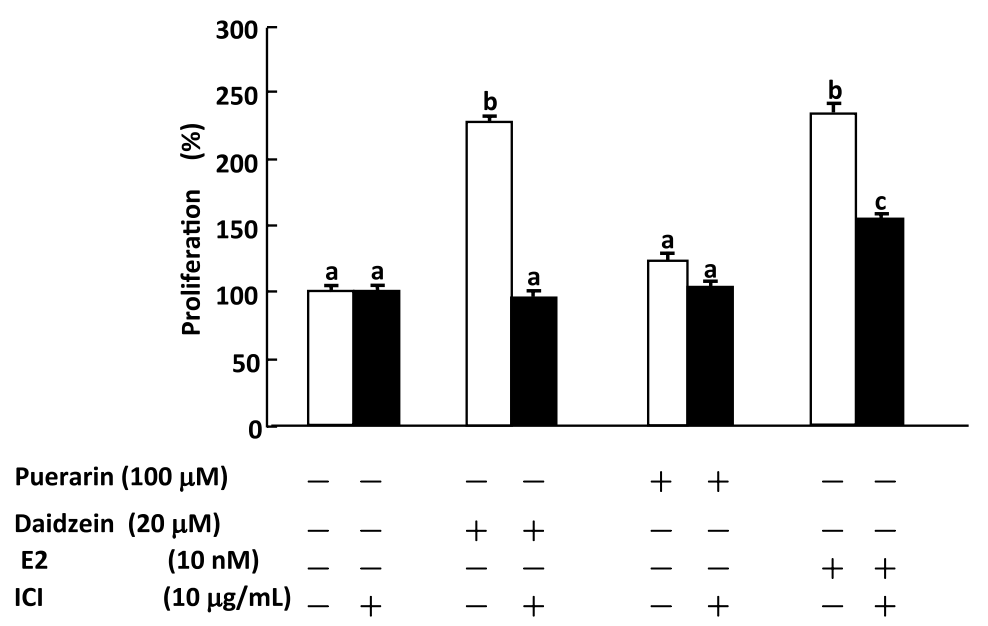

Fig. 7. Effect of estrogen antagonist ICI on MCF-7. MCF-7 cells were treated independently with puerarin, daidzein, and E2 in the presence and absence of E2 antagonist, ICI. Then, the suppression by ICI of the proliferation of MCF-7 cells in the presence of puerarin, daidzein and E2 was analyzed. The presence and absence of them are indicated by + and - , respectively. a, b $<0.01, \mathrm{~b}, \mathrm{c}<0.01$.

This data clearly indicated that puerarin at this dose did not show any in vivo hypertrophic action, the so called estrogen-like action.

Effect of puerarin and ER antagonist, ICI on proliferation of MCF-7 cells

To examine at the cellular level whether puerarin exerts any estrogen-like action, or not, the proliferative action of puerarin on a human breast cancer cell line, MCF-7, was explored. Treatment of MCF-7 cells with puerarin up to $100 \mu \mathrm{M}$ for $4 \mathrm{~d}$ resulted in no enhanced proliferation without cell death, but $10 \mathrm{nM}$ E2 stimulated twice the growth of MCF-7 cells (Fig. 6). To further examine whether puerarin associates with ERs, MCF-7 cells were treated with puerarin, daidzein, and E2 in the presence and absence of an E2 antagonist, ICI. As shown in Fig. 7, puerarin did not enhance the proliferation of MCF-7 cells, but daidzein and E2 enhanced the proliferation twice over the control (no addition). ICI abolished the enhanced action of daidzein completely and of E2 less effectively. Puerarin addition had no effect on MCF-7 cell growth either in the presence or absence of ICI. This finding indicated that puerarin didn't support proliferation of MCF-7 cells, which express ERs, thus showing E2-dependent growth. These data strongly suggest that a puerarin diet does not raise the risk of breast cancer or uterine cancer.

Binding of puerarin to estrogen receptors

Finally, we analyzed the binding profile of puerarin to $\mathrm{ER} \alpha$ and $\beta$. Figure 8 shows the concentration-dependent binding of puerarin, daidzein and E2 to ER $\alpha$ (Fig. $8 \mathrm{~A}$ ) and $\beta$ (Fig. 8B). Puerarin completely lacked the ability to bind to $\operatorname{ER} \alpha$, but showed an extremely weak binding to ER $\beta$, and the binding power to both ERs did not increase when the puerarin concentration was raised up to $100 \mu \mathrm{M}$. ER $\beta$ binding of puerarin was too low to compare with that of E2. On the other hand, daidzein bound to ERs more strongly than puerarin did, and at a concentration of $100 \mu \mathrm{M}$, the affinity of daidzein to both ERs was similar to E2. However, when the
(A)

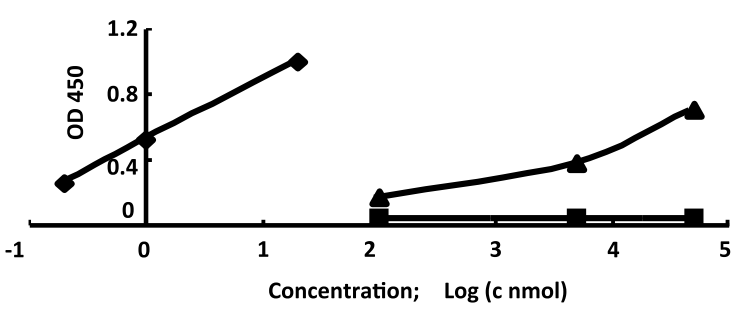

(B)

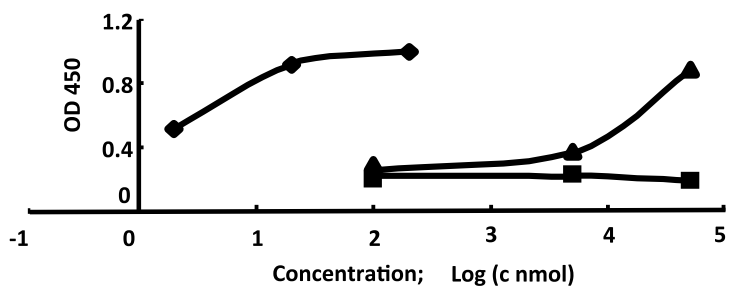

Fig. 8. Binding assay of puerarin to $\operatorname{ER} \alpha$ and $\beta$. The binding of puerarin, daidzein and $\mathrm{E} 2$ to $\mathrm{ER} \alpha(\mathrm{A})$ and $\beta$ (B) were examined with an ELISA assay system by changing the concentration of the ligands. Concentrations of $\mathrm{E} 2$, puerarin and daidzein were from $0.2 \mathrm{nM}$ to $20 \mathrm{nM}$ for E2 and from $100 \mathrm{nM}$ to $2.5 \mathrm{~mm}$ (puerarin and daidzein). Closed rhombus: E2, closed square: puerarin, closed triangle: daidzein.

concentration showing the maximum affinity was compared between daidzein and E2, daidzein bound to both ERs with affinity 5,000-10,000-fold less than E2 did. These data indicated that puerarin $\operatorname{did}$ not bind ER $\alpha$ and $\beta$ or, if it did, bound extremely weakly, compared with daidzein and $\mathrm{E} 2$.

\section{DISCUSSION}

We previously reported that diets supplemented with Kudzu (Pueraria lobata) root and vine extracts improved the bone loss of ovariectomized mice $(14,16)$. These extracts are very rich in puerarin, which constitutes 
more than $50 \%$, sometimes $70 \%$, of the total isoflavonoids. Thus, puerarin is the most probable candidate for the bone loss-preventing action of Kudzu. In the present study, then, we examined the bone loss-preventing action of a puerarin diet in OVX mice. At first, we set a dose of $5 \mathrm{mg}$ puerarin $/ \mathrm{kg}$ b.w./d, which was estimated from the effective dose of our previous feeding study of a Kudzu root and vine diet (16). The puerarin diet at this dose for $8 \mathrm{wk}$ clearly decreased the level of the urinary bone metabolic markers (Fig. 2). Consistent with this, the femur trabecular structure of the puerarin-fed group was restored (Fig. 3). Both results demonstrated that a puerarin diet at this dose prevented bone loss in OVX mice. To learn whether this bone loss-preventing action by puerarin is due to the action of bone formation or absorption, the bone metabolic markers in the serum were measured. The puerarin diet significantly decreased the bone absorption marker TRAP activity, but the level of the bone formation marker osteocalcin remained unchanged. These data clearly indicated that puerarin acted on the bone absorption system, possibly on osteoclasts. These results are in good concordance with the previous Kudzu root and vine experiments (14, 16) which proved that both the extracts selectively suppressed only the TRAP activity but not the bone alkaline phosphatase (another bone formation marker) accompanying the suppressed differntiation of osteoclasts morphologically and the declined expression of the nuclear factor of activated $\mathrm{T}$ cell c1 (NFATc1) expression. Concerning how a puerarin diet improves bone status in OVX mice, the results that the puerarin diet did not restore the morphology or weight of uterus and oviduct, which dramatically diminished after the OVX operation (Fig. 5), suggested that puerarin exhibited no or very little estrogenic action. This consideration was strongly supported by the lack of the enhanced growth of MCF-7 cells by puerarin (Fig. 6) and the lack of effect of the estrogen antagonist ICI on MCF-7 growth in the presence of puerarin (Fig. 7). Furthermore, the results of the in vitro binding profile of puerarin, daidzein and E2 (Fig. 8) clearly demonstrated that puerarin did not associate with $\operatorname{ER} \alpha$ and bound extremely weakly with $\operatorname{ER} \beta$, whereas daidzein bound weakly with $\operatorname{ER} \alpha$ and $\operatorname{ER} \beta$ at low concentrations, but moderately at high concentrations. These data support the conclusion that the bone loss-preventing action of puerarin is not exerted through an ER-mediated pathway. If so, how does puerarin express the action?

Several previous studies have examined the physiology of puerarin in vitro and in vivo. These include protection of animal cells from oxidative stress (17), amelioration of learning and memory defects by long-term puerarin treatment in OVX mice (18), and improved glucose utilization by puerarin in streptozotocininduced diabetic rats (19). We speculated that these three beneficial actions and the present bone loss-preventing action of puerarin might share the same action mechanism. The most possible explanation is due to its antioxidative action, since intracellular oxidative stress universally occurs in various physiological processes leading to diabetes, hypertension, and worsened bone metabolism. In fact, we previously demonstrated that Kudzu root ethanol extracts depressed TRAP gene expression of an osteoclast, the RAW264.7 cell (14), by downregulating NFATc1 through interfering with nuclear translocation of nuclear factor $-\kappa \mathrm{B}(\mathrm{NF}-\kappa \mathrm{B})$, an oxidation responsive factor. $\mathrm{Hu}$ et al. also reported that puerarin attenuated oxidative stress via suppression of an oxidation responsive factor NF- $\kappa \mathrm{B}$ activation in lipopolysaccharide-induced RAW264.7 cells (20). With an animal experiment, Prasain et al. (21) also observed that non-metabolized puerarin and a small amount of its glucuronide were predominantly absorbed and detected in serum of puerarin-fed rats at the early stage of feeding. Recently, we detected puerarin in the bone marrow of puerarin-fed mice (unpublished data). Therefore, it may be a reasonable speculation as the probable mechanism that the suppression of ovariectomy-induced bone loss by puerarin occurs through a non-ER mediated pathway such as modification of the anti-oxidative NF- $\kappa \mathrm{B}$ signal pathway by puerarin in the bone marrow.

Taken all together, it will be appropriate to conclude that puerarin exerts its bone absorption-preventing action through non ER pathways, although the precise mechanism remains for further investigation.

\section{Acknowledgments}

This work was partly supported by the Local Collective Research and Development Program of Nara (2006-2010) from Japan Science and Technology Agency (JST).

\section{REFERENCES}

1) Chien KR, Karsenty G. 2005. Longevity and lineages: toward the integrative biology of degenerative diseases in heart, muscle, and bone. Cell 120: 533-544.

2) Delmas PD. 2002. Treatment of postmenopausal osteoporosis. Lancet 359: 2018-2026.

3) Raisz LG. 2005. Pathogenesis of osteoporosis: concepts, conflicts, and prospects. J Clin Invest 115: 3318-3325.

4) Rodan GA, MartinTJ. 2000. Therapeutic approaches to bone diseases. Science 289: 1508-1514.

5) Teitelbaum SL. 2007. Osteoclasts: what do they do and how do they do it? Am J Pathol 170: 427-435.

6) Riggs BL, Hartmann LC. 2003. Selective estrogenreceptor modulators - mechanisms of action and application to clinical practice. N Engl J Med 348: 618-629.

7) Leclercq G, Heuson JC. 1979. Physiological and pharmacological effects of estrogens in breast cancer. Biochim Biophys Acta 560: 427-455.

8) Kuiper GG, Lemmen JG, Carlsson B, Corton JC, Safe SH, van der Saag PT, van der Burg B, Gustafsson JA. 1998. Interaction of estrogenic chemicals and phytoestrogens with estrogen receptor beta. Endocrinology 39: 42524263.

9) Setchell KD. 1998. Phytoestrogens: the biochemistry, physiology, and implications for human health of soy isoflavones. Am J Clin Nutr 68: 1333S-1346S.

10) Uesugi T, Toda T, Tsuji K, Ishida H. 2001. Comparative study on reduction of bone loss and lipid metabolism abnormality in ovariectomized rats by soy isoflavones, 
daidzin, genistin, and glycitin. Biol Pharm Bull 24: 368372.

11) Lee YB, Lee HJ, Kim KS, Lee JY, Nam SY, Cheon SH, Sohn HS. 2004. Evaluation of the preventive effect of isoflavone extract on bone loss in ovariectomized rats. Biosci Biotechnol Biochem 68: 1040-1045.

12) Gu L, House SE, Prior RL, Fang N, Ronis MJ, Clarkson TB, Wilson ME, Badger TM. 2006. Metabolic phenotype of isoflavones differs among female rats, pigs, monkeys, and women. J Nutr 136: 1215-1221.

13) Ishimi $Y$, Arai $\mathrm{N}$, Wang $\mathrm{X}, \mathrm{Wu} \mathrm{J}$, Umegaki $\mathrm{K}$, Miyaura $\mathrm{C}$, Takeda A, Ikegami S. 2000. Difference in effective dosage of genistein on bone and uterus in ovariectomized mice. Biochem Biophys Res Commun 274: 697-701.

14) Michihara S, Suzuki S, Tang H, Moriyama T, Kawamura Y. 2008. Kudzu (Pueraria lobata) extracts depress bone absorption of ovariectomized mouse by downregulating NFATc1 of osteoclast. J Clin Biochem Nutr 43: 141-144.

15) Ishida $H$, Uesugi $T$, Hirai $K$, Toda $T$, Nukaya $H$, Yokotsuka K, Tsuji K. 1998. Preventive effects of the plant isoflavones, daidzin and genistin, on bone loss in ovariectomized rats fed a calcium-deficient diet. Biol Pharm Bull 21: 62-66.

16) Tanaka T, Tang H, Yu F, Michihara S, Uzawa Y, Zaima N,
Moriyama T, Kawamura Y. 2011. Kudzu (Pueraria lobata) vine ethanol extracts improve ovariectomyinduced bone loss in female mice. J Agric Food Chem 59: 13230-13237.

17) Hwang YP, Jeong HG. 2008. Mechanism of phytoestrogen puerarin-mediated cytoprotection following oxidative injury: ER-dependent up-regulation of PI3K/Akt and HO-1. Toxicol Appl Pharmacol 233: 371-381.

18) Xu X, Hu Y, Ruan Q. 2004. Effects of puerarin on learning-memory and amino acid transmitters of brain in ovariectomized mice. Planta Med 70: 627-631.

19) Hsu FL, Liu IM, Kuo DH, Chen WC, Su HC, Cheng JT. 2003. Antihyperglycemic effect of puerarin in streptozotocin-induced diabetic rats. J Nat Prod 66: 788-792.

20) Hu W, Yang X, Zhe C, Zhang Q, Sun L, Cao K. 2011. Puerarin inhibits iNOS, COX-2 and CRP expression via suppression of NF- $\kappa \mathrm{B}$ activation in LPS-induced RAW264.7 macrophage cells. Pharmacol Rep 63: 781789.

21) Prasain JK, Peng N, Moore R, Arabshahi A, Barnes S, Wyss JM. 2009. Tissue distribution of puerarin and its conjugated metabolites in rats assessed by liquid chromatography-tandem mass spectrometry. Phytomedicine 16: $65-71$. 